\title{
Detailed Computation of Air-Conditioning Modality within Chimpanzee Nasal Airways
}

\author{
Kaouthar Samarat ${ }^{1, *}$, Sho Hanida ${ }^{2}$, Shigeru Ishikawa ${ }^{3}$, Kazunori Kotani ${ }^{4}$ and Teruo \\ Matsuzawa ${ }^{4}$ \\ ${ }^{1}$ Ishikawa Resin Industry Co., Ltd., Ta 1-8 Utani, Kaga-shi, Ishikawa 922-0312, Japan; ${ }^{2}$ Kanazawa \\ Institute of Technology, 7-1 Ohgigaoka, Nonoichi-shi, Ishikawa 921- 8501, Japan; ${ }^{3}$ Kanazawa \\ Municipal Hospital, 3-7-3 Heiwamachi, Kanazawa-shi, Ishikawa 921-8105, Japan; ${ }^{4} J a p a n$ Advanced \\ Institute of Science and Technology, 1-1 Asahidai, Nomi-shi, Ishikawa 923-1292, Japan
}

\begin{abstract}
Nasal cavity of primates and other animals has been reported in many research works. Lots of the previous studies focused on the nasal airflow but few of them considered the internal nasal air-conditioning phenomena. In this study, we investigated the air-conditioning modality within an anatomically accurate computer nasal airways model of a healthy female adult chimpanzee. The finite volumes method was used to compute a steady laminar inspiratory flow with physiological flow rate of $1.4 \mathrm{~m} /$ $\mathrm{s}$, mimicking breathing at rest state. Detailed distributions of air-conditioning in lower, middle and upper airways of the left and right nasal cavities were investigated. The numerical simulation revealed that air-conditioning pattern inside the chimpanzee nasal cavity varies depending to the nasal topology.
\end{abstract}

Keywords: Chimpanzee nasal cavity; Computational fluid dynamics; Nasal air-conditioning.
${ }^{*}$ Correspondence to Author:

Kaouthar Samarat

Ishikawa Resin Industry Co., Ltd., Ta 1-8 Utani, Kaga-shi, Ishikawa 922-0312, Japan;

Email: s1521006@jaist.ac.jp

Tel.: +80-(0)76-151-1231

How to cite this article:

Kaouthar Samarat, Sho Hanida, Shigeru Ishikawa, Kazunori Kotani, Teruo Matsuzawa.Detailed Computation of Air-Conditioning Modality within Chimpanzee Nasal Airways. International Journal of Biomedical Engineering Research, 2018, 2:6

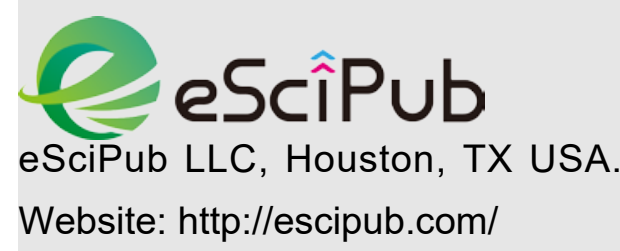




\section{Introduction}

The nasal cavity is the most superior part of the respiratory tract. Its function comprises air exchange, air-conditioning, filtration, vocalization, and sense of smell [1]. The nasal cavity, characterized by thin airway channels, does not allow direct measurements of flow patterns inside the nose. The difficulties involved with experiments led into investigation of the nasal airflow field by means of computational fluid dynamics (CFD). The recent developments in medical imaging, three-dimensional (3D) geometrical modeling and numerical mathematics as well as computer science has released various possibilities for physically realistic numerical simulations of nasal airflow based on computer models of the nasal cavity.

So far, airflow in the nasal cavity has been studied in several kinds of mammals, such as humans [2,3], monkeys [4,5], dogs [6,7], rabbits [8], and rats $[9,10]$.

Recently, there has been an increase in the number of CFD simulations involving realistic nasal cavities of Old World monkeys [11,12] and chimpanzee [12]. [11] investigated the contribution of the maxillary sinus to airconditioning performance, using CFD simulations based on 3D topological computer models, derived from computed tomography (CT) scans, of the nasal cavity of four Japanese macaques, two rhesus macaques, and a savanna monkey. [12] used a CFD model to compare nasal air-conditioning principles and performance in humans, chimpanzees, and macaques; based on digital topological models of the nasal passage reconstructed from medical imaging of six human volunteers, four chimpanzees, four Japanese macaques, and two rhesus macaques.

Even though [12] carefully evaluated nasal airconditioning performance among the three genera (human, chimpanzee, and Old World monkeys) by simulating varied ambient atmospheric conditions, they do only investigated details of air-conditioning distributions occurring in the middle meatus which may not represent what actually occurs in lower and upper airways.

The aim of this research work is to investigate detailed patterns of air-conditioning into the chimpanzee nasal cavity and to discuss the role of anatomical features of the nasal topology in heat and water transfer. Numerical simulation revealed that air-conditioning distribution varies with the transverse airways localization. Whereas similar CFD analyses performed in the same subject compared the air-conditioning performance of human, chimp, and macaque [12], here we reveal further distributions using a CFD model reliable for small-bodied chimpanzee which has a thinner mucosal layer than human. Studying a range of chimp specimens is presently considered beyond the scope of this paper.

\section{Materials and Methods}

\subsection{Anatomical data}

The CT scanning used in this study consisted of 401 slices, spanned from mouth tip to just anterior to larynx, of the head of an adult female chimpanzee (estimated age: 35-year-old; weight: $44.1 \mathrm{~kg}$ ), Pan troglodytes reared at the Primate Research Institute, Kyoto University (KUPRI), Inuyama, Japan [13]. Each slice was composed of $512 \times 512$ pixels in the DICOM format. Scans resolution was $0.351 \mathrm{~mm} / \mathrm{pixel}$ and scanning pitch was $0.5 \mathrm{~mm} / \mathrm{slice}$. The scans were performed in three different axial positions. The CT scans are available on the digital morphology museum (DMM) database (http://dmm.pri.kyoto-u.ac.jp), of the Primate Research Institute, Kyoto University, Inuyama, Japan (CT scans id: PRICT \# 468).

\subsection{Reconstruction of nasal topology}

Black areas of CT scans represented nasal airways. They were extracted, based on a threshold of brightness using Avizo 7 (FEI Visualization Sciences Group, Hillsboro, $\mathrm{OH}$, USA), thus voxel data of the nasal cavity were reconstructed. After converting the voxel data into STereoLithography (STL) data, they were 
modified to derive a 3D smooth surface using Magics 9.5 (Materialize Inc., Leuven, Belgium). Finally, an unstructured tetrahedron mesh was generated using Gambit 2.4 (ANSYS Inc., Canonsburg, PA, USA). The computational mesh contained about $3.7 \times 10^{6}$ tetrahedron cells, $7.6 \times 10^{6}$ triangles, and $7.4 \times 10^{5}$ nonduplicated nodes. Its surface area and total volume were $185.41 \mathrm{~cm}^{2}$ and $41.64 \mathrm{~cm}^{3}$, respectively [14].

\subsection{Computational model of the nasal airflow}

We used an airflow model with heat and humidity transport [15], adapted to reflect chimpanzee respiratory physiology. The governing equations were resolved with the aid of FLUENT (ANSYS Inc., Canonsburg, PA, USA) using the finite volumes scheme.

\subsection{Boundary conditions}

Boundary conditions used to obtain the results in this paper were implemented in a manner similar to that stated by [16]. Physiologically realistic estimates of respiratory rate and tidal volume were calculated based on the body weight of the subject as reported by $[17,18]$.

\subsection{Computational parameters}

Simulation of a steady state airflow inside the chimpanzee nasal cavity during inhalation was performed using the commercial CFD software FLUENT (ANSYS Inc., Canonsburg, PA, USA). Numerical computation was performed with implicit time stepping and a pressure-based solver. The pressure-velocity coupling was accomplished using the SIMPLE method. Green-Gauss Cell Based gradient option was employed to determine spatial gradient. Second Order and Quick discretization schemes were employed for the pressure and the other remaining variables respectively. The computation was run on an Appro PC Cluster (24 core Large Memory Node Appro 1143H) consisting of AMD Opteron 2.6 GHz CPU running the Redhat Linux operating system with 128 GB memory. Parallel computation was performed using the parallelization tool of FLUENT. Convergence was obtained after less than 250 iterations done on 6 processors, taking a total wall-clock time of around 2.5 hours. A norm of nodal energy between iterations of less than $10^{-6}$ was used as the convergence criterion for the termination of energy iterations; other residuals were set to less than $10^{-5}$ except for the water mass fraction which was set to less than $10^{-3}$. The under-relaxation factors were kept as default. Graphical displays of the resulting computational mesh and CFD simulation were generated using Avizo 7.0.

\section{Results}

CFD simulation was performed using standard ambient atmospheric conditions i.e., the temperature of inhaled air was $25^{\circ} \mathrm{C}$ at a relative humidity of $35 \%$.

Figure 1 shows 3D views of temperature distribution within chimpanzee nasal passages (Inhaled air: Temperature $=25{ }^{\circ} \mathrm{C}$, relative humidity $=35 \%$ ). The left panel (A-B-C) of contour plans are sagittal sections of the right nasal passage outbounding from the middle of the right nasal airways (top) to outward (down). The right panel (D-E-F) of contour plans are sagittal sections of the left nasal passage outbounding from the septal side (top) to the middle of the left nasal airways (down). There is no relation between the locations of the sagittal sections in the right and left panels.

The temperature increases slowly within the nasal vestibule region but changes increasingly through the central nasal passages. The inspiratory air was warmed plenty to near body temperature $\left(34{ }^{\circ} \mathrm{C}\right)$ by reaching the posterior margin of the nasopharynx region. Furthermore, the tendency observed for warming the inhaled air in various sagittal sections is different. The rate of warming the inhaled air decreases while approaching the sagittal medial portion of the right nasal passage (Figure1. C-A) and the sagittal medial portion of the left nasal passage (Figure1. D-F). The airflow temperature is higher near the nasal walls. Indeed, the temperature of inspiratory air was adjusted by heat transfer through nasal epithelial layer. This explains the fact that inhaled air temperature increases faster 
near nasal airways lining. The higher tissue-side temperature $\left(34{ }^{\circ} \mathrm{C}\right)$ allows a quick heat exchange with the airflow passing close to nasal cavity walls.
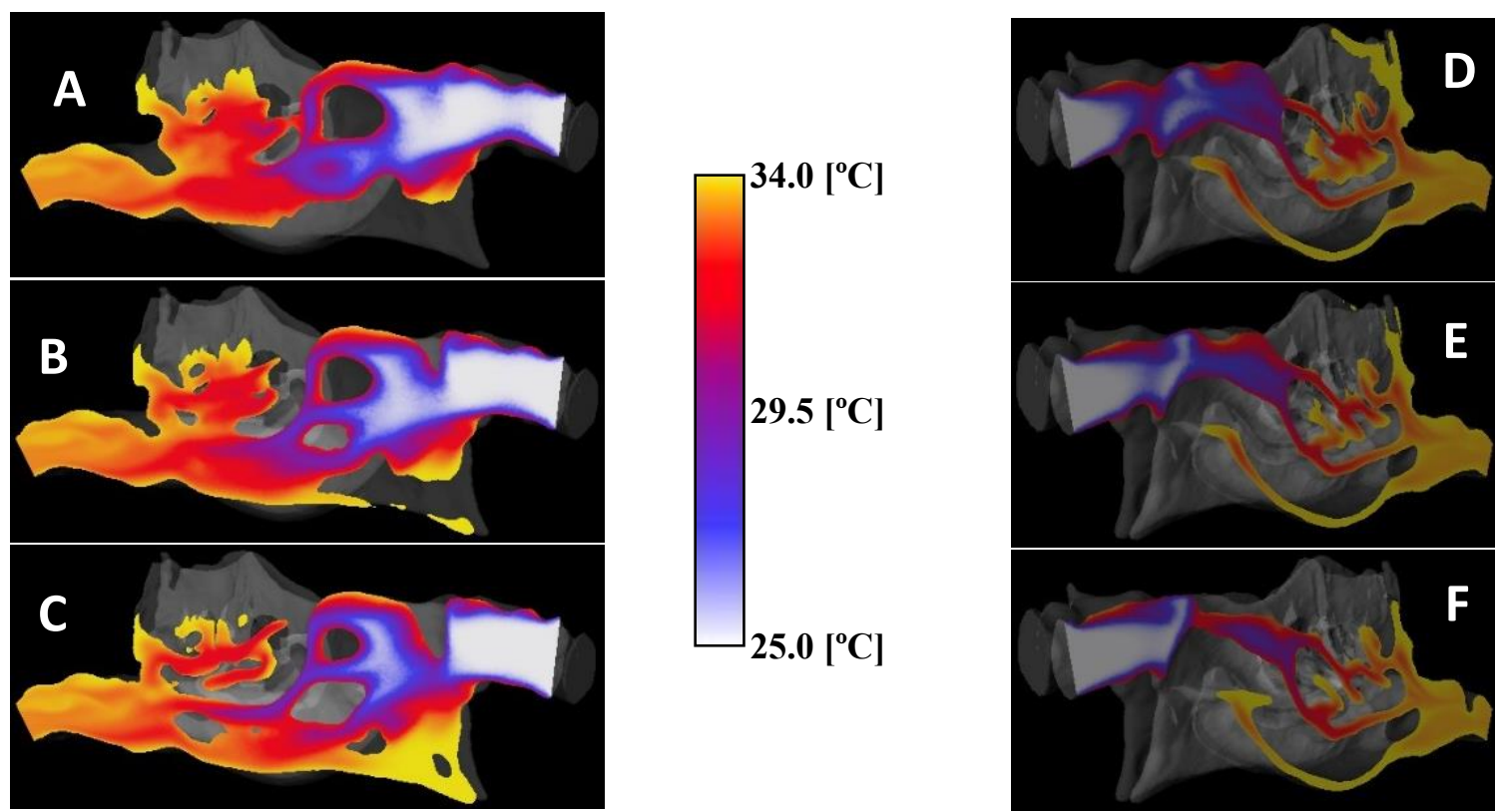

Figure 1. Temperature distribution inside the chimp nasal right (left panel) and left (right panel) passages.
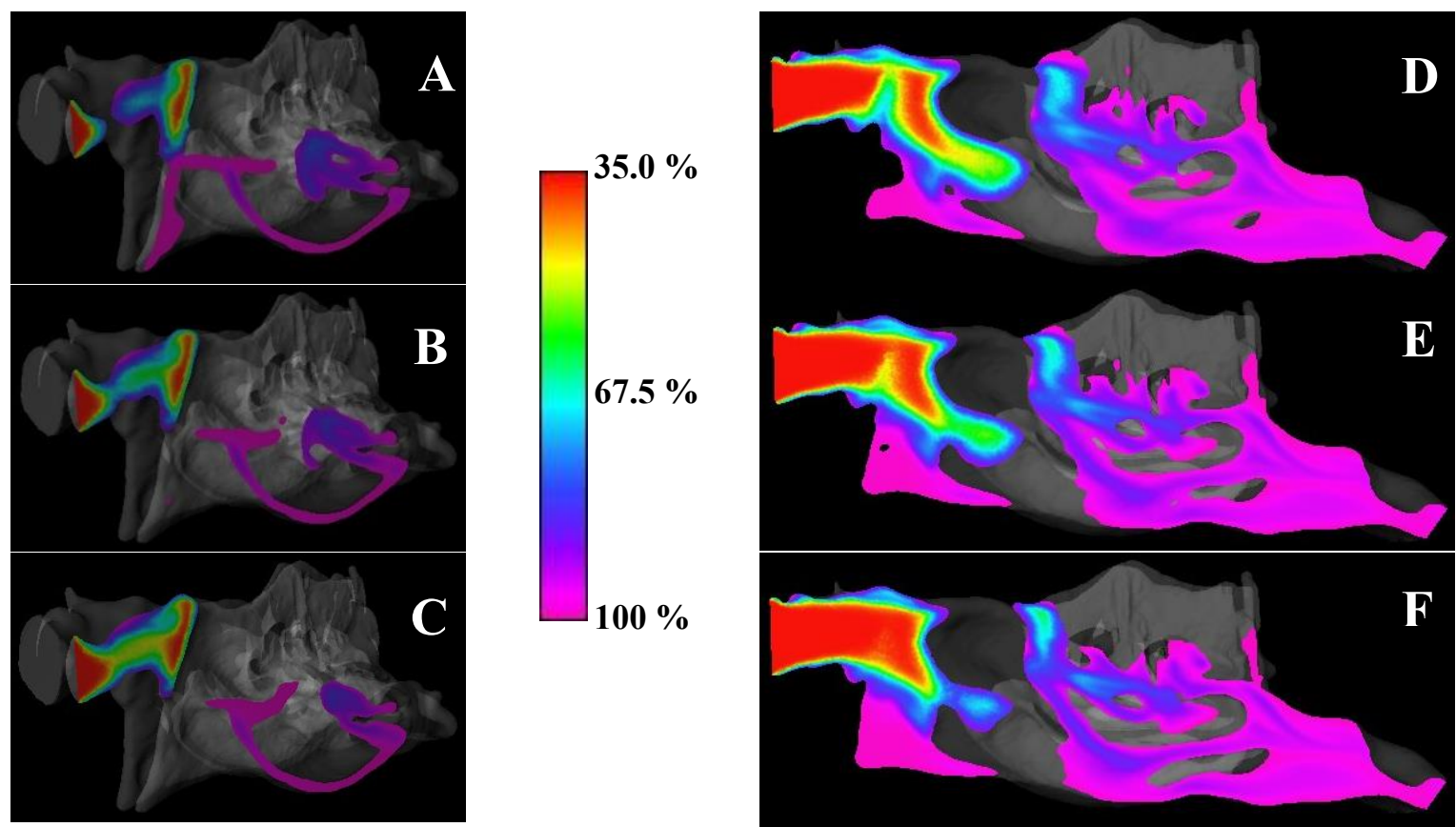

Figure 2. Humidity distribution inside the chimp left nasal passage.

Figure 2 shows 3D views of relative humidity nasal passage outbounding from septal side distribution within chimpanzee left nasal (top) to the middle of the left nasal airway passage (Inhaled air: Temperature $=25{ }^{\circ} \mathrm{C}, \quad$ (down). The right panel (D-E-F) of contour plans relative humidity $=35 \%)$. The left panel $(A-B-C)$ are sagittal sections of the left nasal passage of contour plans are sagittal sections of the left inbounding from outward (top) to the middle of 
the left nasal airway (down). There is no relation between the locations of the sagittal sections in the right and left panels.

The relative humidity increases slowly within the nasal vestibule region but changes increasingly through the central nasal passages. The inspiratory air got adequately moistened to roughly $100 \%$ before reaching the posterior margin of nasopharynx region. In addition, the tendency of humidifying the inhaled air observed in the various sagittal plans is different. The rate of humidifying the inhaled air decreases while approaching the sagittal medial portion of the left nasal passage (Figure1. A-C) and (Figure1. D$F)$. Airflow relative humidity is higher near the nasal walls. Indeed, moistness of inspiratory air got adjusted as a result of water transfer through the nasal epithelial layer. Hence inhaled air's relative humidity increased faster near the nasal airways lining. High tissue-side relative humidity $(100 \%)$ allows a quick exchange of water with the airflow passing close to nasal cavity walls.

\section{Discussion}

Initial temperature of the inspiratory air was less than the body temperature. The inhaled air got gradually warmer while approaching the nasopharyngeal region by absorbing heat from nasal tissue layer via the nasal epithelial layer. By reaching the nasopharynx, the air, initially inspired at $25^{\circ} \mathrm{C}$, was heated to approximately the nasal tissue layer's temperature, $34^{\circ} \mathrm{C}$.

Initially inhaled air had a relative humidity equals $35 \%$, whereas humidity in the nasal passage lining is $100 \%$. The air got moistened by absorbing water from the nasal tissue layer via the nasal epithelial layer. The inhaled air got gradually humidified while approaching the nasopharyngeal region. By reaching the nasopharynx, the dry inspiratory air was moistened to almost the nasal tissue layer's relative humidity, $100 \%$.

Similar points relative to the right and left nasal cavities were chosen for temperature and relative humidity measurements (Figure 3 ). Asymmetry between the right and left nasal cavities is a general aspect common at least for all mammals.

Results showed similarities in temperature and humidity patterns in the lower, middle, and upper airways between right and left nasal passages, respectively. Following graphs of temperature and humidity distributions are for one representative nasal passage (Temperature: left nasal cavity; Humidity: right nasal cavity), because both cavities showed comparable airconditioning modalities. Here, we do report the data which appeared more readable.

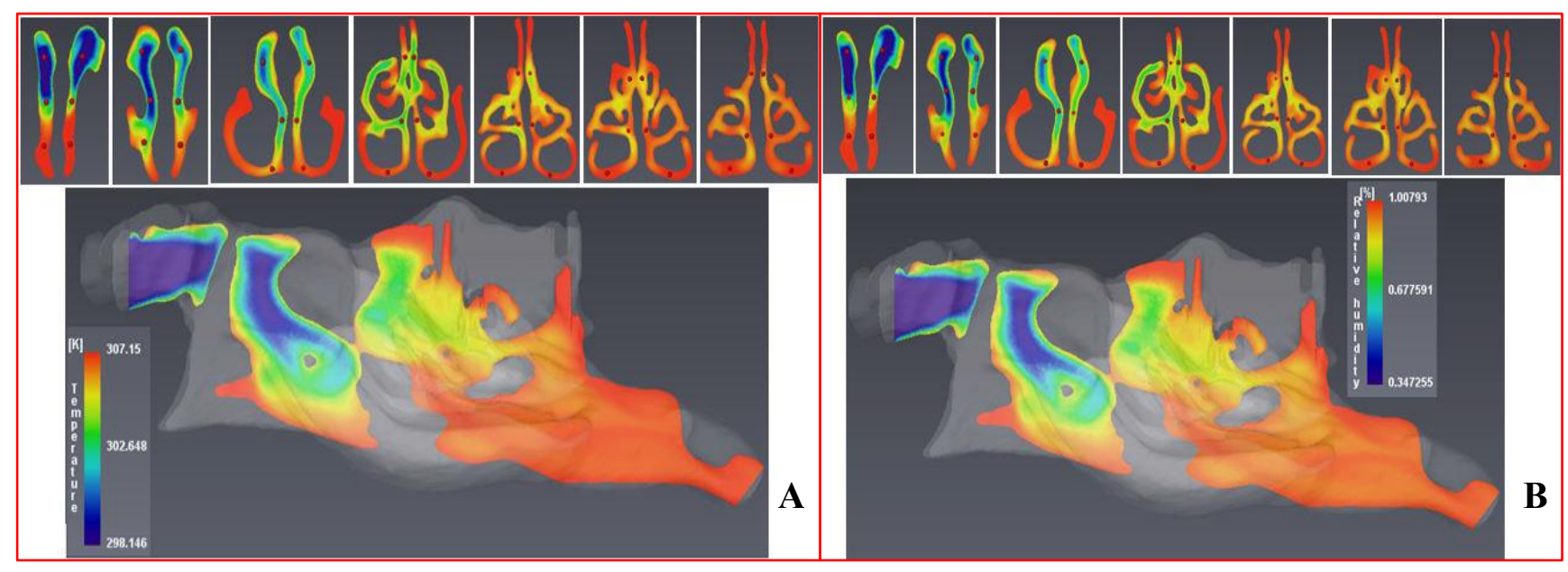

Figure 3. Red dots in coronal cross sections are locations for temperature $(A)$ and relative humidity (B) measurements. 


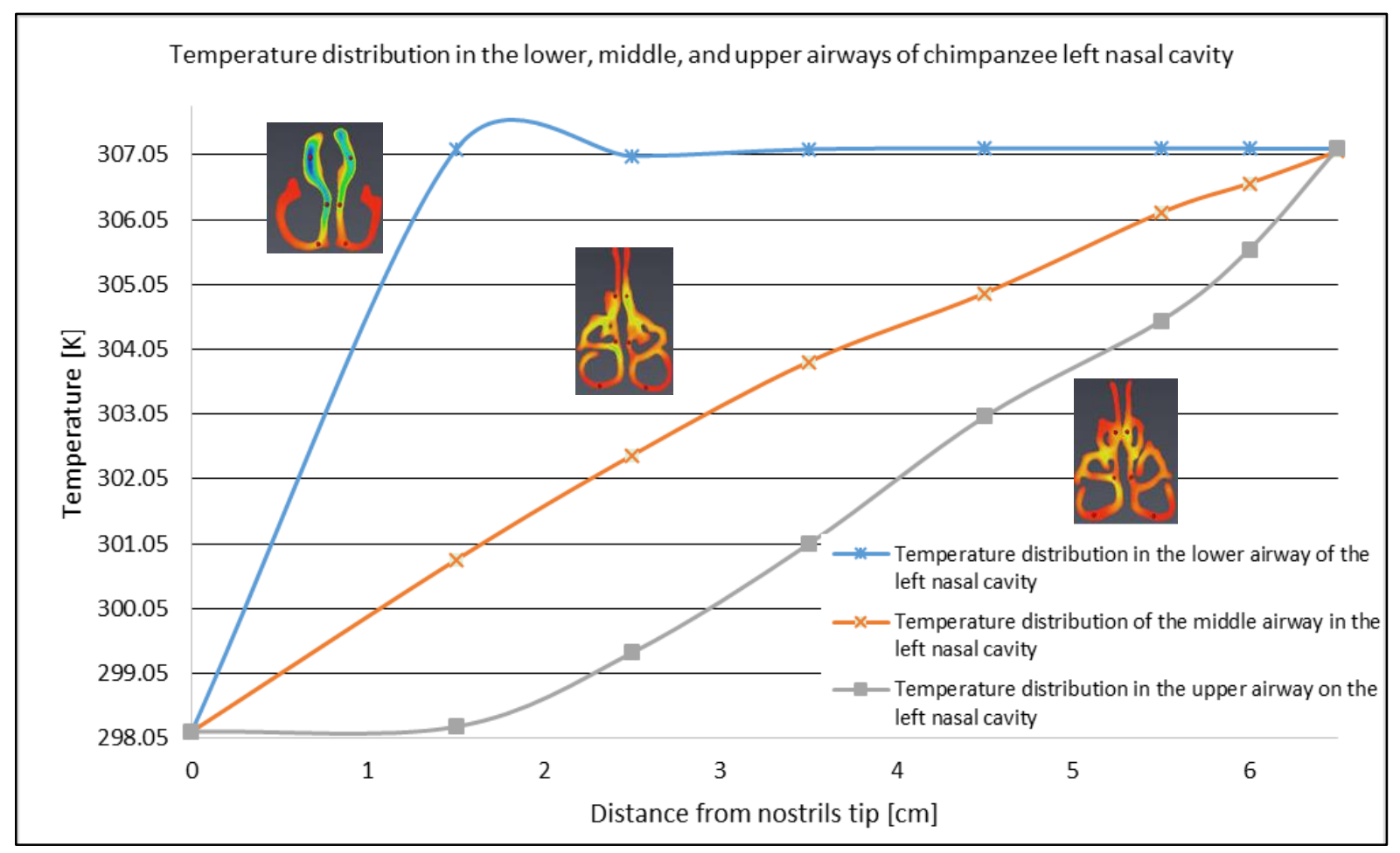

Figure 4. Temperature distributions inside chimpanzee nasal cavity: Temperature distribution in the lower airway of the left nasal cavity (blue dots); Temperature distribution in the middle airway of the left nasal cavity (orange crosses), and temperature distribution in the upper airway of the left nasal cavity (grey squares).

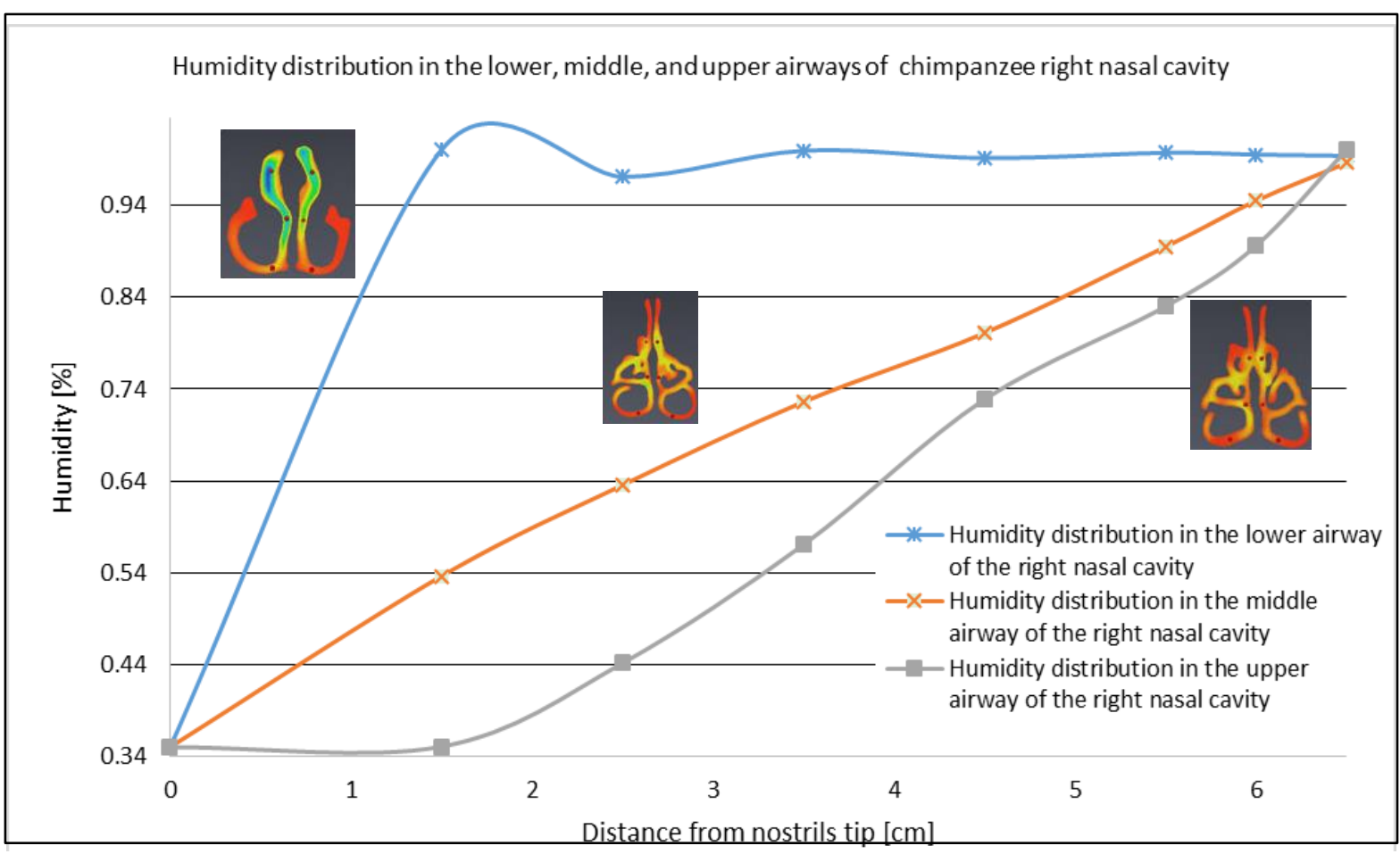

Figure 5. Humidity distributions inside chimpanzee nasal cavity: Humidity distribution in the lower airway of the right nasal cavity (blue dots); Humidity distribution in the middle airway of the right nasal cavity (orange crosses), and humidity distribution in the upper airway of the right nasal cavity (grey squares).

IJBER:http://escipub.com/international-journal-of-biomedical-engineering-research/ 
Temperature and humidity adjustments of inspiratory air behave differently in each transversal site of the nasal airways.

Graphs in Fig.4 and Fig.5 show temperature distribution and humidity distribution in lower, middle, and upper airways of the left and right nasal passages, respectively. In both graphs, the horizontal axe represents the distance from nostrils tip [in $\mathrm{cm}$ ]. The vertical axes represent the temperature $\left[{ }^{\circ}{ }^{\circ} \mathrm{K}\right]$ and the relative humidity [\%], respectively. Humidity and temperature have similar patterns through the nasal cavity.

Temperature's and humidity's patterns of the lower airway may be approached by a rotational curve; i.e. the inhaled cooler and dryer air flowing through the lower airway of the nasal passage tended to get warmed and moistened at once within short distance from the inlet. The respective curves showed that after reaching quickly a maximum, the temperature and humidity decreased slightly and then maintained to the body temperature and humidity values while increasing distance from the nares. The reason behind such structure of inhaled air conditioning is the protrusion of the anterior portion of the inferior turbinate into this airway which created curved lateral walls thus enhancing air-conditioning when surrounded by mucosal nasal lining. The inferior turbinate is the largest [19], spanning nearly the entire length of the main nasal passage.

Temperature and humidity patterns in the middle airway followed a logarithmic curve [12], the constant conditioning of the inhaled air was sustained by the consecutive turbinates' protrusion inside the nasal cavity (Inferior, middle, and superior turbinates respectively).

Unlike the lower airway, in the upper airway the inhaled air kept the same initial temperature and humidity for a while before starting to get warmer and moistened following a linear curve. This airconditioning delay is due to the fact that the superior turbinate, main feature to condition the inhaled air in the upper airways appears only in the latter portion of the main nasal passage and is only about half the length of the middle turbinate.

Reaching same level close to nasopharynx, the temperature and humidity distributions have highest values in the lower airway and lowest values in the upper airway. However, both right and left nasal cavities merge together into a narrow region forming the nasopharyngeal cavity, where the three transversal distributions would be mixed together.

Acknowledgments: The authors thank $T$. Nishimura for providing the CT scans used in this study, K. Kumahata for providing the original source code of CFD method, and F. Mori for his technical support.

\section{References}

1. Cole, P. Modification of inspired air. In The nose: upper airway physiology and atmospheric environment, Proctor, D., Andersen, I., Eds.; Elsevier Biomedical Press: Amsterdam, Netherlands, 1982; pp. 350-375, ISBN 9780444803771.

2. Riazuddin, V. N.; Zubair, M.; Abdullah, M. Z.; Ismail, R.; Shuaib, I. L.; Abdulhamid, S.; Ahmad, K. A. Numerical study of inspiratory and expiratory flow in a human nasal cavity. J Med Biol Eng 2010, 31, pp. 201-206, DOI 10.5405/jmbe.781.

3. Zubair, M.; Riazuddin, V. N.; Abdullah, M. Z.; Ismail, R.; Shuaib, I. L. Airflow inside the nasal cavity-visualization using computational fluid dynamics. Asian Biomed 2010, 4, pp. 657-661.

4. Kepler, G. M.; Richardson, R. B.; Morgan, K. T.; Kimbell, J. S. Computer Simulation of Inspiratory Nasal Airflow and Inhaled Gas Uptake in a Rhesus Monkey. Toxicol. Appl. Pharmacol. 1998, 150, pp. 1-11, DOI 10.1006/taap.1997.8350.

5. Kimbell, J. S.; Subramaniam, R. P.; Gross, E. A.; Schlosser, P. M.; Morgan, K. T. Dosimetry Modeling of Inhaled Formaldehyde: Comparisons of Local Flux Predictions in the Rat, Monkey, and Human Nasal Passages. Toxicological Sciences 2001, 64, pp. 100-110, DOI 10.1093/toxsci/64.1.100

6. Craven, B. A.; Patterson, E. G.; Settles, G. S. The fluid dynamics of canine olfaction: unique nasal 
airflow patterns as an explanation of macrosmia. J R Soc Interface 2010, 7, pp. 933-943, DOI 10.1098/rsif.2009.0490

7. Lawson, M. J.; Craven, B. A.; Paterson, E. G.; Settles, G. S. A computational study of odorant transport and deposition in the canine nasal cavity: implications for olfaction. Chem Senses 2012, 37, pp. 553-566, DOI 10.1093/chemse/bjs039.

8. Corley, R. A.; Minard, K. R.; Kabilan, S.; Einstein, D. R.; Kupart, A. P. Magnetic resonance imaging and computational fluid dynamics (CFD) simulations of rabbit nasal airflows for the development of hybrid CFD/PBPK models. Inhal Toxicol 2009, 21, pp. 512-518, DOI 10.1080/08958370802598005.

9. Yang, G. C.; Scherer, P. W.; Mozell, M. M. Modeling inspiratory and expiratory steady-state velocity fields in the Sprague-Dawley rat nasal cavity. Chem Senses 2007, 32, pp. 215-223, DOI 10.1093/chemse/bjl047.

10. Zehong, W.; Zhixiang, X.; Bo, L.; Fuqiang, X. Numerical simulation of airway dimension effects on airflow patterns and odorant deposition patterns in the rat nasal cavity. PLoS ONE 2013, 8, DOI 10.1371/journal.pone.0077570.

11. Mori, F.; Hanida, S.; Kumahata, K.; MiyabeNishiwaki, T.; Suzuki, J.; Matsuzawa, T. Minor contributions of the maxillary sinus to the airconditioning performance in macaque monkeys. $J$ Exp Biol. 2015, 218, pp. 2394-2401, DOI 10.1242/jeb.118059.

12. Nishimura, T.; Mori, F.; Hanida, S.; Kumahata, K.; Ishikawa, S. Impaired Air Conditioning within the Nasal Cavity in Flat-Faced Homo. PLoS Comput Biol 2016, 12, DOI 10.1371/journal.pcbi.1004807.

13. Matsuzawa, T., Tomonaga, M., \& Tanaka, M. (Eds.). Cognitive development in chimpanzees; Springer: Tokyo, Japan, 2006; ISBN 978-4-43130246-9.

14. Samarat, K., Matsuzawa, T. A Computational Model of the Anatomy of Realistic Chimpanzee Nasal Airways. IJIR 2016, 2, pp. 357-364.

15. Kumahata, K.; Mori, F.; Ishikawa, S.; Matsuzawa,

T. Nasal flow simulation using heat and humidity models. J Biomech Sci Engineer 2010, 5, pp. 565-577, DOI 10.1299/jbse.5.565.

16. Samarat, K.; Kumahata, K.; Hanida, S.; Nishimura, T.; Mori, F.; Ishikawa, S.; Matsuzawa, T. Application of computational fluid dynamics to simulate a steady airflow in all regions of chimpanzee nasal cavity. In Procedia Engineering, Proceedings of the 25th International Conference on Parallel Computational Fluid Dynamics, Changsha, China, May 20-24; Kenli Li, Matthew Smith, Mariano Vazquez (Eds.); Elsevier Ltd.: 2013; pp. 264-269.

17. Worthington, J.; Young, I. S.; and Altringham, J. D. The relationship between body-mass and ventilation rate in mammals. J. Exp. Biol. 1991, 161, pp. 533-536.

18. Stahl, W. R. Scaling of respiratory variables in mammals. J. Appl. Physiol. 1967, 22, pp. 453-460.

19. House, E. L., Pansky, B., Jacobs, M. S., \& Wagner, B. M. (1966). Gross structure of the ear, nasal cavity and paranasal sinuses of the Chimpanzee. Anatomical Record, 155(1), 77-88. https://doi.org/10.1002/ar.1091550109 